\title{
Three-Dimensional Micromagnetic Simulation of Spatial Distribution of Magnetization in Thick Cobalt Layers
}

\author{
M. Kisielewski ${ }^{a, *}$, A. MAZIEWski ${ }^{a}$ AND V. ZABLOtskiI ${ }^{b}$ \\ ${ }^{a}$ Faculty of Physics, University of Białystok, Lipowa 41, 15-424 Białystok, Poland \\ ${ }^{b}$ Institute of Physics ASCR, Na Slovance 2, 18221 Prague, Czech Republic
}

\begin{abstract}
Spatial magnetization distribution of cobalt layer is studied by means of three-dimensional micromagnetic simulations in the range of cobalt thickness $d$ from 21 to $249 \mathrm{~nm}$. In this range, a spin-reorientation phase transition occurs, while the cobalt thickness increases, from a state with in-plane magnetization, to a state with out-of-plane components of magnetization. An infinite cobalt layer is modelled by the $750 \mathrm{~nm} \times 750 \mathrm{~nm} \times d$ structure consisting of cubic cells of size of $3 \mathrm{~nm}$ and the periodic boundary conditions. For larger thicknesses, a labyrinth, partially closed, stripe structure has been found.
\end{abstract}

DOI: 10.12693 /APhysPolA.127.520

PACS: $75.40 . \mathrm{Mg}, 75.60 . \mathrm{Ch}, 75.70 . \mathrm{Ak}, 75.70 . \mathrm{Kw}$

\section{Introduction}

Magnetization states and magnetization processes in cobalt single layers as well as multilayers, have been intensively studied recently [1] as one of the most interesting subject of the physics of magnetism, which additionally has a huge application potential (e.g. in the engineering of high-density storage devices). Among others, a large efforts have been focused on the study of the spinreorientation phase transitions, driven by the thickness of cobalt layer. There are two such transitions: one undergoes at the Co thickness of $c a .2 \mathrm{~nm}$, the second one - at ca. $20 \mathrm{~nm}$. In the first case, magnetization changes from an out-of-plane state into an in-plane state, as the thickness increases [2]. In the second case, magnetization rises from an in-plane state, back to a state with out-of-plane components, while the thickness increases [3]. Systematic study of this case by means of micromagnetic simulations, were reported in Ref. [4], where one of the most interesting results was the formation of a closed stripe domain structure with Bloch domain walls, the whole resembling a set of vortex with in-plane oriented vortex axis. These studies had been performed using a low-time-consuming, two-dimensional mode of micromagnetic simulation [5], where the three-dimensional (3D) sample was modelled as one layer of elongated simulation cells. The present paper is aimed to a development of these studies and uses the $3 \mathrm{D}$ mode of simulation, as well as more advanced version of the software. Of course, the base of the new approach is today available computers, which have both a huge calculation power and memory capacity, and make it possible to simulate large structures within relatively short time.

* corresponding author; e-mail: kisiel@uwb.edu.pl

\section{Simulations details}

Micromagnetic simulations have been performed using a fresh version of the OOMMF software [5], namely the $1.2 \mathrm{a} 5$ version issued in 2012. The 3D-simulation mode has been used. An infinite cobalt layer (laying in the $x y$-plane) with a $d$ thickness (measured along the $z$-axis) has been modelled by the structure of sizes of $750 \mathrm{~nm} \times 750 \mathrm{~nm} \times d$, composed of cubic cells of size of $3 \mathrm{~nm}$ (i.e. smaller than exchange length equal to $3.2 \mathrm{~nm}$ in the case of cobalt), and the periodic boundary conditions in both $x$ and $y$ directions - see Fig. 1. Consequently, the total number of cells has been varied from 437500 in the case of $d=21 \mathrm{~nm}$, to 5187500 in the case of $d=249 \mathrm{~nm}$.

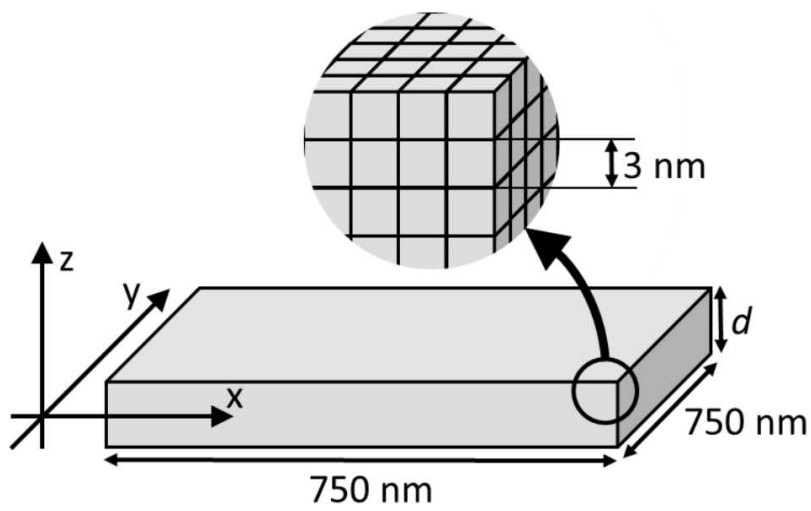

Fig. 1. Coordinate system and configuration of cells of a simulated sample.

Every time, a sinusoidal-like magnetization distribution has been set as a simulation starting distribution of magnetization, by means of the following dependence of the $m_{z}$ normalized magnetization component on the $x$ coordinate: $m_{z}(x)=0.75 \cos (2 \pi x / p)$, where $p$ is period, and with $m_{x}=0$ and $m_{y}>0$ for the all cells. For a given $d$, simulations with starting distributions characterized by different $p$ have been performed in the aim 
A)

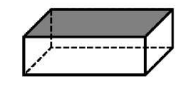

B)

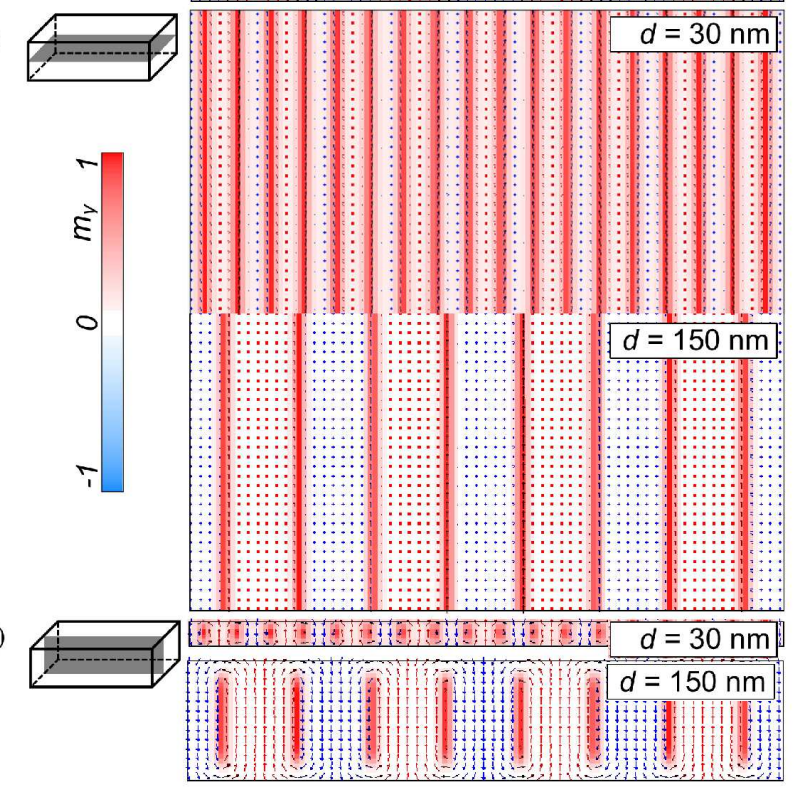

Fig. 2. Final spatial distributions of magnetization, corresponding to the lowest total magnetic energy, in the case of both $d=30 \mathrm{~nm}$ and $d=150 \mathrm{~nm}$ and the following layers of the sample (see additional diagrams at the top of each figure showing the localization of each visualized layer): (A) top layer parallel to the $x y$-plane (this image is composed of parts of distributions obtained for the two thicknesses, as is indicated in the image); (B) the middle layer parallel to the $x y$-plane (a composed image, too); (C) cross-section layer parallel to the $x z$-plane in the middle of the sample. Each arrow represents magnetization vector averaged over the cube of $4^{3}$ cells. In Fig. $2 \mathrm{~A}$, in the case of $d=150 \mathrm{~nm}$, one can distinguish Néel walls, which are parallel to the $y$-axis. In Fig. 2B one can see either arrowheads or arrow-ends as red and blue points, respectively. Additionally, coloured shadow indicates the $m_{y}$ component of magnetization (see the scale shown at the left side of Fig. 2B). Here $m_{y}$ is larger than zero $\left(m_{y}>0\right)$ in the all sample, thus it is marked in red. In Fig. $2 \mathrm{~B}$ one can see Bloch walls in the form of straight, red-shadowed thick lines oriented along the $y$-axis. In Fig. $2 \mathrm{C}$, for the case of $d=150 \mathrm{~nm}$, one can see the areas of existence of Bloch walls across the thickness of the sample in the form of red-shadowed, elongated-stadium-like shapes parallel to the $z$-axis.

to find the final distribution with the lowest total magnetic energy. As in our previous simulations, the following magnetic parameters have been used: saturation magnetization $M_{\mathrm{s}}=1420 \mathrm{kA} / \mathrm{m}$ and exchange constant $A=1.3 \times 10^{-11} \mathrm{~J} / \mathrm{m}$. The thickness dependent first uniaxial anisotropy constant $K_{1}(d)$ for a given $d$ has been taken from Ref. [6] for the case of Co layer sandwiched between gold layers, i.e. $K_{1}$ varied from $0.684 \mathrm{MJ} / \mathrm{m}^{3}$ in the case of $d=21 \mathrm{~nm}$ down to $0.634 \mathrm{MJ} / \mathrm{m}^{3}$ in the case of $d=249 \mathrm{~nm}$.

\section{Results}

In the all cases of simulated samples with different values of $d$, the final spatial distribution of magnetization is found to be of the type of a partially closed stripe domain structure with the Bloch domain walls - see an example shown in Fig. 2, where different cross-sections of the simulated sample with both $d=30 \mathrm{~nm}$ and $d=150 \mathrm{~nm}$ are visualized in the aim to clarify the distributions as much as possible.

For different $d$ value, a different $p$ have been found to have the lowest energy. The equilibrium $p(d)$ dependence is shown in Fig. 3.

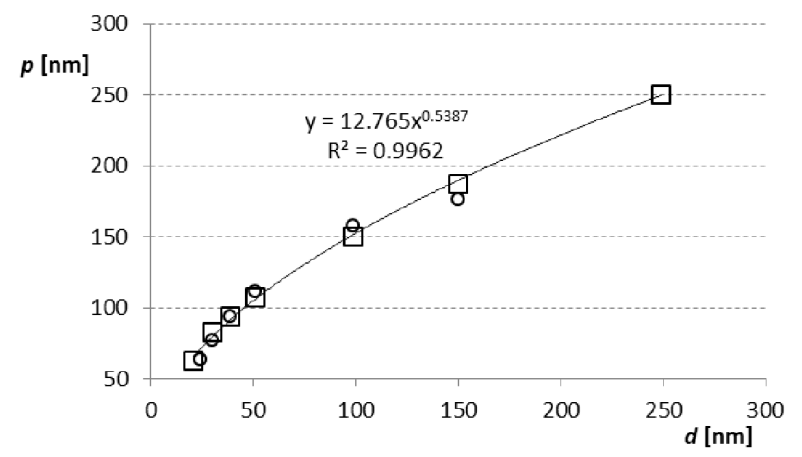

Fig. 3. Equilibrium $p(d)$ dependence obtained in the 3D mode of simulation (squares). Solid line represents the best fitting to these points using a power function with parameters written in the image, as well as the $R^{2}$ parameter describing the quality of the fitting. For comparison, the results of simulation obtained in the two-dimensional mode reported in Ref. [4], are added (circles).

During simulation for larger thicknesses, a very interesting spatial magnetization distribution in the form of labyrinth, partially closed, stripe structure has appeared accidentally, see an example shown in Fig. 4. It should be underlined that such distribution of magnetization does not correspond to the lowest energy, thus must be considered as a metastable state [7]. Usually, simulation has come to such a solution, when the number of period of sinusoidal-like domains at the beginning of simulation was very low, e.g. was equal to 2 . In Fig. 4 one can find very interesting singularities of the magnetization distribution, which can be interpreted as Bloch lines. 


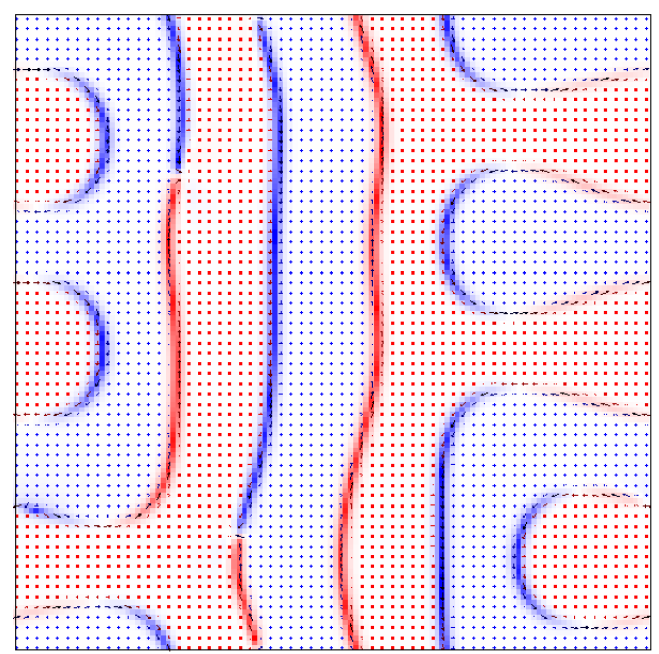

Fig. 4. Metastable spatial distribution of magnetization in the case of $d=249 \mathrm{~nm}$ and the middle layer of the sample, parallel to the $x y$-plane (just like in Fig. 2B). As in Fig. 2B, one can see either arrowheads or arrowends (as red and blue points, respectively) in the volume of domains. Domain walls are clearly visible by means of shadowed curves indicating the $m_{y}$ component of magnetization. Now $m_{y}>0$ is marked in red while $m_{y}<0$ - in blue (see the scale shown in Fig. 2B). A sudden change of the colour of the shadow, when one is moving along a wall, indicates a Bloch line.

\section{Discussion}

The $p(d)$ dependence shown in Fig. 3 can be accurately described using an almost square root function. The square root dependence is commonly used for describing experimental results in this range of Co thicknesses [3], as well as it is theoretically confirmed by the model of partially closed domain structure [8].

If one intends to perform simulations for more thick samples, in the aim to obtain first a wavy structure and next the domain branching phenomenon (and creation of dagger-like domains), one should take into account the following limitations. Experimental results reported in Ref. [9] show that in the case of cobalt, the wave structure starts to appear at $d \approx 2000 \mathrm{~nm}$. For this thickness, period is expected to be $p \approx 760 \mathrm{~nm}$. To simulate this case, it would be necessary to increase the width of the simulate sample at least twice, and to increase the thickness eight times, in respect of the largest structure simulated recently, i.e. $750 \mathrm{~nm} \times 750 \mathrm{~nm} \times 249 \mathrm{~nm}$. Consequently, the total number of cells of simulated structure would increase from 5 to 80 million. Unfortunately, this seems to be too high for today computers and it would extend the time of calculation unendurably.

\section{Conclusions}

Spatial distribution of magnetization of an infinite cobalt layer of the thickness in the range from tens to hundreds nm can be successfully modelled using the 3D mode of the OOMMF software with the periodic boundary conditions. This approach opens new possibilities of study of equilibrium and metastable 3D magnetization distributions and allows revealing singularities of magnetization distributions as e.g. Bloch lines.

\section{Acknowledgments}

This work was supported by the Foundation for Polish Science Team Programme co-financed by the EU European Regional Development Fund, OPIE 20072013. V.Z. acknowledges the financial support of the Czech Science Foundation (GACR) under project 1318993S. This research was supported in part by PL-Grid Infrastructure.

\section{References}

[1] A. Maziewski, J. Fassbender, J. Kisielewski, M. Kisielewski, Z. Kurant, P. Mazalski, F. Stobiecki, A. Stupakiewicz, I. Sveklo, M. Tekielak, A. Wawro, V. Zablotskii, Phys. Status Solidi A 211, 1005 (2014).

[2] M. Kisielewski, A. Maziewski, T. Polyakova, V. Zablotskii, Phys. Rev. B 69, 184419 (2004).

[3] M. Hehn, S. Padovani, K. Ounadjela, J.P. Bucher, Phys. Rev. B 54, 3428 (1996).

[4] M. Kisielewski, A. Maziewski, V. Zablotskii, J. Magn. Magn. Mater. 316, 277 (2007).

[5] M. Donahue, D. Porter, http://math.nist.gov/oommf.

[6] M. Kisielewski, A. Maziewski, M. Tekielak, A. Wawro, L.T. Baczewski, Phys. Rev. Lett. 89, 87203 (2002).

[7] V.A. Zablotskii, K.V. Lamonova, Y.A. Mamalui, Physica B 205, 371 (1995).

[8] R. Szymczak, Electron Techn. 1, 5 (1968).

[9] A. Hubert, R. Schäfer, Magnetic Domains, SpringerVerlag, Berlin 1998. 